\title{
PROFINITE EXTENSIONS OF CENTRALIZERS AND THE PROFINITE COMPLETION OF LIMIT GROUPS
}

\author{
PAVEL ZALESSKII AND THEO ZAPATA
}

\begin{abstract}
We introduce and investigate a class of profinite groups defined via extensions of centralizers analogous to the extensively studied class of finitely generated fully residually free groups, that is, limit groups (in the sense of Z. Sela). From the fact that the profinite completion of limit groups belong to this class, results on their group-theoretical structure and homological properties are obtained.
\end{abstract}

\section{INTRODUCTION}

In the last decades, limit groups have been attracting the attention of many mathematicians (e.g., [CG05], [AB06], [BH07], [CZ07], [Wil08], [Koch10], [BK17]), specially because they played a fundamental role in the recent solution ([Sel01] et seq. and [KhM98a] et seq.) of the outstanding Tarski problem on free groups.

Two basic group-theoretical reasons for so much attention are the following: limit groups are approximable by free groups, according to their many definitions (cf. [Sel01], [BBau67], [Rem89], [KhM98a]); and, limit groups constitute a class of groups that envelopes free groups of finite rank and free abelian groups of finite rank. Limit groups also have many interesting properties. Some of their most simple properties are: being torsion-free, having infinite abelianization (if the group is non-trivial), that solubility implies commutativity, being centerless, and being commutativity-transitive (cf. [CG05, Prop. 1]).

Our present work introduces and studies a class of profinite groups $\mathcal{Z}$, defined via extensions of centralizers analogous to limit groups.

A profinite extension of a centralizer is a free profinite amalgamated product $G \amalg_{C} A$, where $A$ is a finitely generated free abelian profinite group, $C$ is a procyclic direct factor of $A$, and $N_{G}(H)=C$ for every non-trivial closed subgroup $H$ of $C$. The class $\mathcal{Z}$ consists of all finitely generated closed subgroups of profinite groups obtained by finitely many iterated extensions of centralizers starting from a free profinite group of finite rank (see Section 3 for details).

The following results are proved in sections 3 and 4.

Theorem A. Let $G$ be a profinite group in the class $\mathcal{Z}$. Then $G$ has finite cohomological dimension and hence is torsion-free; furthermore, if its

Date: May 15, 2018.

2010 Mathematics Subject Classification. Primary 20E18; Secondary 20E06, 20E08, 20J05. 
cohomological p-dimension $c d_{p}(G)$ is $\geq 2$ then $c d_{p}(G)=\max \left\{2, \alpha_{p}(G)\right\}$, where $\alpha_{p}(G)$ is the supremum of the $c_{p}(A)$ with $A$ running over the abelian closed subgroups of $G$. In particular, this gives a uniform (finite) bound for the rank of abelian subgroups of $G$.

The next theorem is a statement of a Tits alternative flavour.

Theorem B. Let $G$ be a profinite group in the class $\mathcal{Z}$. Then $G$ has no nonabelian free pro-p closed subgroup for each prime $p$ if, and only if, $G$ is abelian or meta-procyclic projective.

We also completely describe the finitely generated pro- $p$ subgroups of groups from class $\mathcal{Z}$.

Theorem C. A pro-p group in the class $\mathcal{Z}$ is a free pro-p product of free abelian pro-p groups.

One of the main properties of limit groups is commutative transitivity. This properties already fails for free profinite groups. The next theorem describes those that are commutativity-transitive, showing that this property for profinite groups is quite restrictive.

Theorem D. A commutativity-transitive profinite group in the class $\mathcal{Z}$ is either pro-p (as described in Theorem C) or abelian.

We prove that the profinite completion of a limit group belongs to the class $\mathcal{Z}$; that gives a source of important examples. Thus all above theorems are true for finitely generated subgroups of the profinite completion of a limit group.

Theorem E. The profinite completion of a limit group belongs to the class $\mathcal{Z}$.

We also establish more specific properties for the profinite completion of limit groups:

Theorem F. Let $G$ be the profinite completion of a limit group. Then:

(a) $G$ is of homological type $\mathrm{FP}_{\infty}$ over $\mathbb{F}_{p}$ and over $\mathbb{Z}_{p}$.

(b) the homological Euler-Poincaré characteristic of $G$ over $\mathbb{F}_{p}$ or $\mathbb{Z}_{p}$ coincides with the homological Euler-Poincaré characteristic $\chi(\Gamma)$ of $\Gamma$ over $\mathbb{Z}$; hence it is always $\leq 0$ and equals 0 if, and only if, $\Gamma$ is abelian.

(c) if $U_{1} \supseteq U_{2} \supseteq U_{3} \supseteq \ldots$ is a descending sequence of open normal subgroups of $G$ with trivial intersection, then

$$
\lim _{k \rightarrow \infty} \frac{\operatorname{dim}_{\mathbb{F}_{p}} \mathrm{H}_{q}\left(U_{k}, \mathbb{F}_{p}\right)}{\left(G: U_{k}\right)}=\lim _{k \rightarrow \infty} \frac{\operatorname{dim}_{\mathbb{F}_{p}} \mathrm{H}^{q}\left(U_{k}, \mathbb{F}_{p}\right)}{\left(G: U_{k}\right)}=\left\{\begin{array}{cl}
-\chi(\Gamma), & \text { if } q=1, \\
0, & \text { otherwise. }
\end{array}\right.
$$


The techniques involved in proving our theorems consist of the profinite version of Bass-Serre theory of groups acting on trees (e.g., [Rib17], [ZM88] and [ZM89]) and homological methods (e.g., [Ser94] and [RZ00b]). The fact that the profinite completion of a limit group belongs to our introduced class, relies on the result established by H. Wilton [Wil08, Thm. B] that limit groups virtually retract onto each of its finitely generated subgroups, and also on a result about centralizers in the profinite completion of limit groups obtained by S. Chagas and P. Zalesskii [CZ07, Lemma 3.5]. Some facts of the combinatorial theory of profinite groups are collected in Section 2.

Theorems A, B, D, and E were proved for our new class of profinite groups in the Ph.D thesis [Zap11, Cap. 2].

In the final section of the paper we comment on group-theoretical properties of groups belonging to the class of limit groups, to the class of pro- $p$ groups studied by Kochloukova and Zalesskii [KZ11], and to the presently defined class of profinite groups via extensions of centralizers.

Notation. Throughout this paper, $p$ is a prime number. The field of $p$ elements is denoted by $\mathbb{F}_{p}$. The additive group of the ring of $p$-adic integers is $\mathbb{Z}_{p}$. If $\pi$ is a set of prime numbers, then $\mathbb{Z}_{\pi}$ denotes the cartesian product $\prod_{p \in \pi} \mathbb{Z}_{p}$; the set of all prime numbers but $p$ is denoted by $p^{\prime}$.

For a subset $A$ of a topological group $G$ we denote by $\langle A\rangle$ the (abstract) subgroup of $G$ generated by $A$ and by $\bar{A}$ the topological closure of $A$ in $G$; the subgroup of $G$ topologically generated by $A$ is $\overline{\langle A\rangle}$. By $d(G)$ we denote the smallest cardinality of a (topological) generating subset of a profinite group $G$. The profinite completion of a discrete group $\Gamma$ is $\widehat{\Gamma}$.

For a profinite group $G$ acting continuously on a space $X$ we denote the point stabilizer for each $x$ in $X$ by $\operatorname{stab}_{G}(x)$.

Henceforth, we use capital greek letters for discrete groups and capital roman letters for profinite groups. The rest of our notation is very standard and basically follows [RZ00b] and [RZ00a].

\section{Preliminary Results}

In this section we collect a few results on the combinatorial theory of profinite groups used in the paper. Further information on this subject can be found in [RZ00b], [RZ00a], and [Rib17]. For the cohomology theory of profinite groups we refer to [Ser94, Ch. I] and [RZ00b, Ch. 6 and 7].

An amalgamated free profinite product $G=G_{1} \amalg_{C} G_{2}$ is proper if the canonical maps $G_{1} \rightarrow G$ and $G_{2} \rightarrow G$ are injective. We shall make use of the fact proved by L. Ribes [Rib71, Thm. 2.3] (cf. [RZ00b, Exerc. 9.2.6]) that an amalgamated free 
profinite product is proper, whenever the amalgamating subgroup is central in one of the amalgamated free factors.

Proposition 2.1 ([RZ96, Cor. 2.7(ii)]). Let $G_{1}$ and $G_{2}$ be profinite groups with a common procyclic subgroup $C$ such that $G=G_{1} \amalg_{C} G_{2}$ is proper. Let $c \in C$. The following relation among normalizers holds, $N_{G}(\overline{\langle c\rangle})=N_{G_{1}}(\overline{\langle c\rangle}) \amalg_{C} N_{G_{2}}(\overline{\langle c\rangle})$.

We assume basic concepts and facts of the profinite version of Bass-Serre theory as developed by Mel'nikov, Ribes, and Zalesskii [ZM88], [ZM89], and [Rib17]; in particular, we only mention here that a profinite tree is an acyclic connected profinte graph. For our purposes we emphasize the following two results. The first is a sort of Tits alternative; the second has no abstract analogue (here the compactness of the tree is essential).

Theorem 2.2 ([Zal90, Thm. 3.1]). Let $G$ be a profinite group acting on a profinite tree $T$. Then one of the following holds:

(a) $G$ stabilizes some vertex of $T$.

(b) $G$ has a non-abelian free pro-p subgroup $P$ such that $P \cap \operatorname{stab}_{G}(v)=\{1\}$, for each vertex $v$ of $T$.

(c) There exists an edge e of $T$, whose stabilizer $\operatorname{stab}_{G}(e)$ is normal in $G$ and $G / \operatorname{stab}_{G}(e)$ is isomorphic to one of the following profinite groups: (c.1) a projective group $\mathbb{Z}_{\pi} \rtimes \mathbb{Z}_{\rho}$, where $\pi \cap \rho=\varnothing$; (c.2) a Frobenius group $\mathbb{Z}_{\pi} \rtimes \mathbb{Z} / m \mathbb{Z}$, where $m$ is not divisible by any prime of $\pi$ and $[k, c] \neq 1$ for each $k \in \mathbb{Z}_{\pi}-\{0\}$ and each $c \in \mathbb{Z} / m \mathbb{Z}-\{0\}$; (c.3) the infinite dihedral pro- $\pi$ group $\mathbb{Z} / 2 \mathbb{Z} \amalg^{\pi} \mathbb{Z} / 2 \mathbb{Z}$ isomorphic to $\mathbb{Z}_{\pi} \rtimes \mathbb{Z} / 2 \mathbb{Z}$, where $2 \in \pi$.

Proposition 2.3 ([Zal90, Lemma 1.5] complemented by [Ser77, Prop. 19]). Let G be a profinite group acting on a profinite tree $T$. There exists a (non-empty) minimal G-invariant profinite subtree $D$ of $T$. Moreover, if $D$ has more than one element, then $D$ is unique and infinite.

In order to prove the main result of the following section, we recall the concept of the Tits straight path (cf. [Tit70, Prop. 3.2, p. 192] or [Ser77, Prop. 24]). Let $X$ be an abstract tree and denote its (canonical) length function by $l$ and its vertex set by $V(X)$. Let $\alpha$ be a fixed-point-free automorphism of $X$. The set of all vertices $v$ of $X$ such that $l(v, \alpha(v))=\inf _{w \in V(X)} l(w, \alpha(w))$ constitute the vertex set of a unique straight path (i.e., a doubly infinite chain) $T_{\alpha}$; this subtree $T_{\alpha}$ is called the Tits straight path of $\alpha$. If $[v, w]$ denotes the unique path joining vertices $v$ and $w$ in a tree and $\left[v, w\left[=[v, w]-\{w\}\right.\right.$, then $T_{\alpha}=\langle\alpha\rangle\left[v, \alpha v\left[\right.\right.$ for any $v$ in $T_{g}$.

For the reader's convenience the next proposition collects fundamental facts in the profinite usage of the Tits straight path ( $c f$. [RZ96, proofs of Lemma 2.8 and Prop. 2.9]; [ZM88, Example 1.20A] and the previous Proposition 2.3; and [RSZ96, proof of Lemma 4.3]). 
Proposition 2.4. Let $\Gamma=A *_{Z} B$ be a free product with 1-generated amalgamation. Assume $\Gamma$ is residually finite, $\Gamma$ induces the profinite topology on $A, B$, and $Z$, and $A, B$, and $Z$ are closed in the profinite topology of $\Gamma$. Let $\gamma$ be an element of $\Gamma$ not belonging to a conjugate of either $A$ or $B$ (in other words, $\gamma$ is hyperbolic). Let $T_{\gamma}$ be the Tits straight path of $\gamma$ in the standard tree $S$ on which $\Gamma$ acts, and let $\overline{T_{\gamma}}$ be its closure in the standard profinite tree $\widehat{S}$ on which $\widehat{\Gamma}$ acts. Then:

(a) $\overline{T_{\gamma}}=\overline{\langle\gamma\rangle}\left[v, \gamma v\left[\right.\right.$ for any $v$ in $T_{\gamma}$;

(b) $\overline{T_{\gamma}}$ is the unique minimal $\overline{\langle\gamma\rangle}$-invariant profinite subtree of $\widehat{S}$;

(c) $T_{\gamma}$ is a connected component of $\overline{T_{\gamma}}$ considered as as abstract graph, in other words, the only vertices of $\overline{T_{\gamma}}$ that are at a finite distance from a vertex of $T_{\gamma}$ are those of $T_{\gamma}$.

\section{Profinite extensions of Centralizers}

Some properties of limit groups immediately pass to their profinite completion. For instance, say $\widehat{\Gamma}$ is the profinite completion of a limit group. If $\widehat{\Gamma}$ is virtually soluble, then it must be abelian; each open subgroup of $\widehat{\Gamma}$ is finitely presented (as a profinite group); the group $\widehat{\Gamma}$ has infinite continuous abelianization if $\Gamma$ is non-trivial.

The present work introduces a class of profinite groups defined via extensions of centralizers analogous to limit groups. We provide some motivation to define such class.

An extension of centralizer of a discrete group $\Gamma$ is an amalgamated free product $\Gamma *_{Z}(Z \times B)$ where $Z$ is the centralizer of an element of $\Gamma$ and $B$ is a free abelian group of finite rank.

In the discrete case, the centralizer of each non-trivial element in a free group is infinite-cyclic and, after performing an extension of centralizer, the centralizer of the element becomes abelian. In the profinite case, the centralizer of each nontrivial element that generates $\widehat{\mathbb{Z}}$ in a free profinite group is meta-procyclic and, after performing an extension of centralizer, the centralizer becomes either meta-abelian, or (non-trivial procyclic)-by-(infinite dihedral pro- $\pi$ ), or contains a non-abelian free pro- $p$ subgroup. See Lemma 4.2 and Theorem 4.3, and Propostition 4.7.

Our construction is now introduced. Let $\mathcal{C}$ be a non-empty class of finite groups closed with respect to taking subgroups, quotients, and finite direct products. We inductively define the following classes of pro- $\mathcal{C}$ groups:

The class $\mathcal{Z}_{0}(\mathcal{C})$ of all free pro-C groups of finite rank.

For each integer $n>0$, the class $\mathcal{Z}_{n}(\mathcal{C})$ of all groups $G_{n}$, such

that $G_{n}$ is the amalgamated free pro-C product $G_{n-1} \amalg_{C_{n-1}} A_{n-1}$ satisfying the following conditions: 
A1. $C_{n-1}$ is a procyclic proper subgroup and a direct factor of a free abelian pro-C group of any finite rank $A_{n-1}$.

A2. $C_{n-1}$ is a closed subgroup of the group $G_{n-1}$ of $\mathcal{Z}_{n-1}(\mathcal{C})$ such that the normalizer in $G_{n-1}$ of each non-trivial procyclic subgroup of $C_{n-1}$ coincides with $C_{n-1}$.

Definition 3.1. The class $\mathcal{Z}(\mathcal{C})$ of pro-C groups consists of all finitely generated profinite subgroups of some group $G_{n}$ of $\mathcal{Z}_{n}(\mathcal{C})$ with $n \geq 0$. When $\mathcal{C}$ consists of all finite groups, we simply denote $\mathcal{Z}_{n}(\mathcal{C})$ by $\mathcal{Z}_{n}$, and $\mathcal{Z}(\mathcal{C})$ by $\mathcal{Z}$.

Note that, when $\mathcal{C}$ consists of all finite $p$-groups the class $\mathcal{Z}(\mathcal{C})$ coincides precisely with the class of groups originally studied by Kochloukova and Zalesskii [KZ11]. Indeed, the property of limit groups that the normalizer and the centralizer of any non-trivial 1-generated subgroup coincide and are abelian holds for such pro- $p$ groups ([KZ11, Thm. 5.1]).

Remark 3.2. It follows from condition $\mathbf{A} 2$ in the definition of the class $\mathcal{Z}_{n}$, that each non-trivial procyclic closed subgroup $P$ of $C_{n-1}$ has abelian normalizer in $G_{n}$. In fact, in light of Proposition 2.1 we have

$$
N_{G_{n}}(P)=N_{G_{n-1}}(P) \amalg_{C_{n-1}} N_{A_{n-1}}(P)=C_{n-1} \amalg_{C_{n-1}} A_{n-1}=A_{n-1} .
$$

The next theorem, whose pro- $p$ analogue is unknown, gives the main source of important examples of groups from the class $\mathcal{Z}$. Before we prove it, recall that a group acts $k$-acylindrically on a graph if only the trivial element fixes pointwise each subset of diameter $>k$. Note that the action of $G_{n}$ on its standard profinite tree is not 1-acylindrical, since two distinct edges $1 C_{n-1}$ and $g C_{n-1}$ incident to the vertex $1 A_{n-1}$ have stabilizers whose intersection is $C_{n-1}$.

Lemma 3.3. For each $n \geq 1$, the action of $G_{n}$ on its standard profinite tree is 2-acylindrical.

Proof. Fix $n$. We shall prove that any three consecutive edges have stabilizers that intersect trivially. After a translation in the profinite tree of $G_{n}$, we obtain two distinct edges $1 C_{n-1}$ and $g C_{n-1}$ incident to the vertex $1 G_{n-1}$; here, $g \in G_{n-1}-C_{n-1}$. Note that $g C_{n-1} g^{-1}$ is not contained in $C_{n-1}$ (indeed, $N_{G_{n-1}}\left(C_{n-1}\right)$ equals $\left\{x \in G_{n-1} \mid x C_{n-1} x^{-1} \subseteq C_{n-1}\right\}$ because closed subsemigroups in compact groups are subgroups, and $N_{G_{n-1}}\left(C_{n-1}\right)=C_{n-1}$ by definition). On the other hand, since $C_{n-1}$ is abelian, $g C_{n-1} g^{-1}$ normalizes $C_{n-1} \cap g C_{n-1} g^{-1}$. So, this intersection must be trivial; otherwise, its normalizer in $G_{n-1}$ would be $C_{n-1}$ by condition A2. The result follows.

Theorem 3.4. The profinite completion of a limit group belongs to the class $\mathcal{Z}$. 
Proof. First, let $\Gamma$ be a finitely generated subgroup of a free group $\Gamma_{0}$. Then $\Gamma$ is a free group and its profinite completion is a free profinite group of finite rank.

Next, let $\Gamma$ be a finitely generated subgroup of the limit group $\Gamma_{n}$ given by $\Gamma_{n}=\Gamma_{n-1} * Z_{n-1} A_{n-1}$, where $Z_{n-1}$ is a 1-generated proper subgroup and a direct factor of a finite rank free abelian group $A_{n-1}$, and $Z_{n-1}$ is self-centralizing in $\Gamma_{n-1}$.

Note that $\widehat{\Gamma_{n}}=\widehat{\Gamma_{n-1}} \amalg_{\widehat{Z_{n-1}}} \widehat{A_{n-1}}$. Indeed, the group $\Gamma_{n}$ is residually free; moreover, by [RZ96, Lemma 2.1], the profinite topology of $\Gamma_{n}$ induces the profinite topology on $\Gamma_{n-1}, A_{n-1}$, and $Z_{n-1}$; so, the stated equality follows (cf. [RZ00b, Ex. 9.2.7(2)]).

Now, we prove that the group $\widehat{\Gamma}$ is naturally embedded in $\widehat{\Gamma_{n}}$. Indeed, Wilton [Wil08, Thm. B] showed that there exists a finite index subgroup $\Upsilon$ in $\Gamma_{n}$ containing $\Gamma$ and there exists a group homomorphism $\rho: \Upsilon \rightarrow \Gamma$ that is an extension of the identity map of $\Gamma$; thus, $\Upsilon$ is isomorphic to $\operatorname{ker}(\rho) \rtimes \Gamma$. By [RZ00b, Lemma 3.1.4(a) and Lemma 3.1.5(a)], the profinite topology of $\Gamma_{n}$ induces the profinite topology on $\Upsilon$ and the profinite topology of $\Upsilon$ induces the profinite topology on $\Gamma$. So, the desired embedding follows ( $c f$. [RZ00b, Lemma 3.2.6]).

To conclude the proof, we shall establish the last requirement of condition A2.

Claim. Let $\Gamma$ be a limit group. If $Z$ is a 1-generated subgroup of $\Gamma$ such that $N_{\Gamma}(Z)=Z$, then $N_{\widehat{\Gamma}}(\overline{\langle g\rangle})=\bar{Z}$ for each non-trivial element $g$ in $\bar{Z}$. (Here the closures are taken in $\widehat{\Gamma}$.)

It suffices to prove the claim for $\Gamma=\Gamma_{n}$. Let $S$ be the standard tree on which $\Gamma_{n}$ acts (if $n=0$, then $S$ is the Cayley graph of $\Gamma_{0}$ ). Let $\zeta$ be a generator of $Z$.

Suppose $\zeta$ does not fix any vertex of $S$ (i.e., the element $\zeta$ is hyperbolic). Fix $n \geq 1$. In order to apply Proposition 2.4, recall that Wilton [Wil08, Thm. A] proved that limit groups are subgroup separable. Let $T_{\zeta}$ be the Tits straight path of $\zeta$ in $S$ and let $\overline{T_{\zeta}}$ be its closure in the standard profinite tree $\widehat{S}$ on which $\widehat{\Gamma_{n}}$ acts.

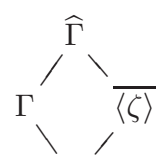

$\langle\zeta\rangle$

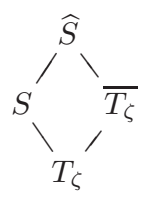

$T_{\zeta}$

Let $N=N_{\widehat{\Gamma}}(\overline{\langle g\rangle})$. By Proposition 2.4(b), $N$ acts naturally on $\overline{T_{\zeta}}$; for, the translation of $\overline{T_{\zeta}}$ by an element of $N$ is a minimal $\overline{\langle\zeta\rangle}$-invariant profinite subtree of $\widehat{S}$. Now, note that $N$ acts freely on $\overline{T_{\zeta}}$. Indeed, if $h$ in $N$ fixes some element of $\overline{T_{\zeta}}$, then, by Proposition 2.4(a), there exists $c$ in $\overline{\langle g\rangle}$ such that $c^{-1} h c$ fixes an element of $T_{\zeta}$; so, by Proposition 2.4(c), $T_{\zeta} \cup c^{-1} h c T_{\zeta}=T_{\zeta}$; whence, $c^{-1} h c$ acts on $T_{\zeta}$. Let $H$ be the set of all elements of $N$ that leave $T_{\zeta}$ invariant; from the 2-acylindrical action of $\widehat{\Gamma}$ on $\widehat{S}$ (Lemma 3.3), the obvious map $H \rightarrow \operatorname{Aut}\left(T_{\zeta}\right)$ is an embedding; so, being $H$ torsion-free (Theorem A) and being $\operatorname{Aut}\left(T_{\zeta}\right)$ the free 
product of two groups of order 2 (i.e., the infinite dihedral group of $\mathbb{Z}$ ), it follows that the automorphism induced by $c^{-1} h c$ is the identity, that is, $h=1$.

Next, we use an argument from covering theory. By Proposition 2.4(a), $N \backslash \overline{T_{\zeta}}$ is a quotient of the finite circuit $\langle\zeta\rangle \backslash T_{\zeta}$; on the other hand, the connected profinite graph $\overline{T_{\zeta}}$ is a simply connected profinite graph, because so is $\widehat{S}$ (cf. [ZM89, Lemma A in 1.3 and Thm. 3.4]); therefore, by [Zal89, Thm. 2.8 and Lemma 2.3] we get that $N$ is isomorphic to the abelian group $\widehat{\mathbb{Z}}$.

So, we obtain

$$
\bar{Z} \subseteq N \subseteq C_{\widehat{\Gamma}}(\bar{Z})=\overline{C_{\Gamma}(Z)}=\overline{N_{\Gamma}(Z)}
$$

where the last two equalities are from [CZ07, Prop. 3.5 and Lemma 3.3].

Note that the proof for $n=0$ is, mutatis mutandis, the same (cf. [RZ96, proof of Lemma 3.4]). This case is done.

Finally, suppose $\zeta$ fixes some vertex of $S$. Then, since it cannot fix a vertex that is a coset of $A_{n-1}$, it is, up to conjugation in $\Gamma_{n}$, an element in $\Gamma_{n-1}$. By [ZM88, Thm 3.12] $N_{\widehat{\Gamma}}\left((\overline{\langle g\rangle})=N_{\widehat{\Gamma}_{n-1}}((\overline{\langle g\rangle})\right.$ so that the claim follows by induction on $n$.

Simple examples of groups in the class $\mathcal{Z}$ are therefore the profinite completion of surface groups, except those of non-orientable surfaces of genus 1,2 , or 3 (i.e., the real projective plane, the Klein bottle, and Dyck's surface).

It is clear that the free profinite product of the profinite completion of two limit groups belongs to the class $\mathcal{Z}$. The next result provides more examples of groups in the class $\mathcal{Z}$.

The important construction of G. Baumslag known, nowadays, as Baumslag double arises in his classical paper [Bau62] on residually free groups. It consists of an amalgamated free product such that the amalgamated free factors are isomorphic groups and the amalgamated subgroup is cyclic and self-normalizing in each amalgamated free factor. Adding an obvious condition we obtain that a Baumslag double of a group in the class $\mathcal{Z}$ stays in the class $\mathcal{Z}$ :

Proposition 3.5. Let $G$ be a finitely generated profinite group contained in some group $G_{n}$ of $\mathcal{Z}_{n}$, let $\varphi: G \rightarrow G^{\varphi}$ be an isomorphism of profinite groups. Assume that $C$ is a procyclic subgroup of $G$ such that for each non-trivial procyclic subgroup $D$ of $C$ we have $N_{G_{n}}(D)=C$. Then the amalgamated free profinite product $G \amalg_{C=C \varphi} G^{\varphi}$ belongs to $\mathcal{Z}_{n+1}$.

Proof. Let $C_{n}=C$ and $A_{n}=C \times \overline{\langle t\rangle}$ with $t$ such that $A_{n}$ is a free profinite abelian group of rank 2 , and define $G_{n+1}=G_{n} \amalg_{C_{n}} A_{n}$. From hypothesis, $G_{n+1}$ belongs to $\mathcal{Z}_{n+1}$. Since $G \amalg_{C=C^{\varphi}} G^{\varphi}$ is isomorphic to the subgroup $\overline{\left\langle G, t G t^{-1}\right\rangle}$ of $G \amalg_{C_{n}} A_{n}$, it is a finitely generated closed subgroup of $G_{n+1}$. 
One may apply this proposition to $G=\widehat{\Gamma_{n}}$ and $C=\bar{Z}$ with $Z$ a 1-generated self-normalizing subgroup of $\Gamma_{n}$ ( $c f$. Claim in the proof of Theorem 3.4). The proof of the proposition carries over in the discrete and pro- $p$ situations.

\section{Main Results}

We begin this section by proving the homological Theorems A and F.

Proof of Theorem A. Say $G$ is a closed subgroup of some group $G_{n}$ of $\mathcal{Z}_{n}$. If $n=0$, we have $c d_{p}(G) \leq 1$. Suppose then $n \geq 1$. In virtue of a Mayer-Vietoris sequence associated to the amalgamated free product $G_{n-1} \amalg_{C_{n-1}} A_{n-1}(c f$. [ZM88, (2.7)]), we obtain

$$
c d_{p}(G) \leq \sup \left\{2, c d_{p}\left(G \cap x G_{n-1} x^{-1}\right), c d_{p}\left(G \cap y A_{n-1} y^{-1}\right) \mid x \in G_{n}, y \in G_{n}\right\},
$$

because $c d_{p}\left(C_{n-1}\right) \leq 1$.

Let $\alpha_{p}(G)=\sup \left\{c d_{p}(A) \mid A \leq_{c} G, A\right.$ abelian $\}$. Then, by induction $c d_{p}\left(G \cap x G_{n-1} x^{-1}\right) \leq \max \left\{2, \alpha_{p}(G)\right\}$; and clearly $c d_{p}\left(G \cap y A_{n-1} y^{-1}\right) \leq \alpha_{p}(G)$. Hence $c d_{p}(G) \leq \max \left\{2, \alpha_{p}(G)\right\}$. Furthermore, if $c d_{p}(G) \geq 2$, then we plainly have $\max \left\{2, \alpha_{p}(G)\right\} \leq c d_{p}(G)$.

To prove Theorem $\mathrm{F}$ we use the fact established by Grunewald, Jaikin-Zapirain, and Zalesskii [GJZ08, Thm. 1.3] that limit groups are good (in the sense of Serre [Ser94, I.§2.6]). A discrete group is good if the Galois cohomology of its profinite completion coincides with the corresponding (discrete) group cohomology for finite discrete modules. More precisely: a discrete group $\Gamma$ is called good if for each finite discrete $\widehat{\Gamma}$-module $M$, the obvious homomorphism

$$
\mathrm{H}^{q}(\widehat{\Gamma}, M) \longrightarrow \mathrm{H}^{q}(\Gamma, M)
$$

is bijective for any $q \geq 0$.

Regarding Theorem A, note that if $G$ is the profinite completion of a limit group $\Gamma$, for each $q>c d(\Gamma)$ we have $\mathrm{H}^{q}(G, M)=0$ for every discrete $G$-module $M$ which is finite, and hence which is torsion. So $c d(G) \leq c d(\Gamma)$.

Lemma 4.1. Let $\Gamma$ be a discrete group and let $M$ be a discrete finite $\widehat{\Gamma}$-module. Consider the direct system (resp., the inverse system) consisting of the restriction maps $\mathrm{H}^{q}(N, M) \rightarrow \mathrm{H}^{q}\left(N^{\prime}, M\right)$ (resp., corestriction maps $\left.\mathrm{H}_{q}\left(N^{\prime}, M\right) \rightarrow \mathrm{H}_{q}(N, M)\right)$, over the indexing set of all finite index normal subgroups $N$ of $\Gamma$. We have:

(i) Goodness of $\Gamma$ implies $\lim _{N} \mathrm{H}^{q}(N, M)=0$ for all $q \geq 1$.

(ii) If $\mathrm{H}_{q}(N, \mathbb{Z})$ is finitely generated for each sufficiently small $N$ for all $q \geq 0$, then $\lim _{N} \mathrm{H}^{q}(N, M)=0$ implies $\lim _{N} \mathrm{H}_{q}(N, M)=0$ for all $q \geq 1$.

Proof. (i) Fix $q$ and $N$. It suffices to prove that for each $x$ in $\mathrm{H}^{q}(N, M)$ there exists a finite index subgroup $N_{x}$ of $N$ such that the image of $x$ under the map 
$\mathrm{H}^{q}(N, M) \rightarrow \mathrm{H}^{q}\left(N_{x}, M\right)$ in the direct system is zero. This is a well-known exercise of Serre [Ser94, I.§2.6, Exerc. 1, p. 13].

(ii) We may and do assume that $M$ is a trivial $N$-module for each sufficiently small $N$; by cofinality this does not alter the limits. Fix $q \geq 1$ and $N$. There exists a finite index subgroup $N^{\prime}$ of $N$ such that the map $\mathrm{H}^{q}(N, M) \rightarrow \mathrm{H}^{q}\left(N^{\prime}, M\right)$ in the direct system is the null map. It suffices to prove that the corestriction $\mathrm{H}_{q}\left(N^{\prime}, M\right) \rightarrow \mathrm{H}_{q}(N, M)$ is the null map.

By the Universal coefficient theorem ( $c f$. [CE56, Thm. 3.3 and Thm. 3.3a of Ch. VI]) we have the following commutative diagrams with splitting exact rows of abelian groups

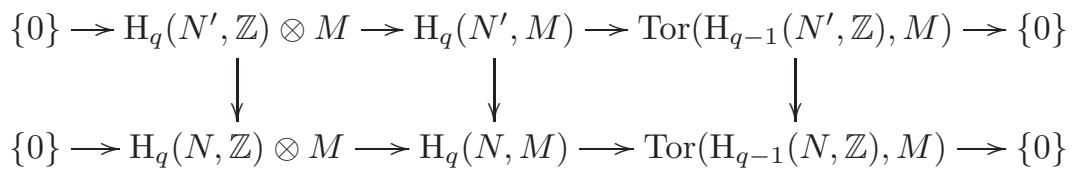

and

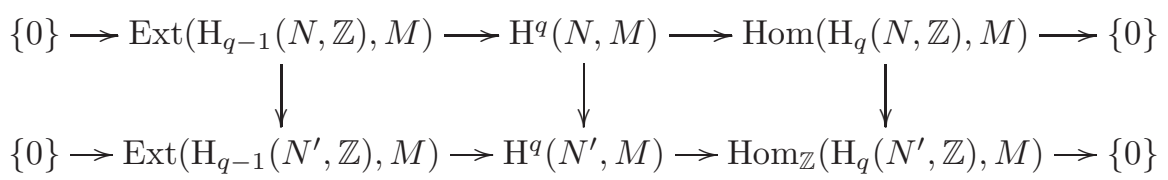

where $\otimes=\otimes_{\mathbb{Z}}$, Tor $=$ Tor $_{1}^{\mathbb{Z}}$, Ext $=$ Ext $_{\mathbb{Z}}^{1}$, and Hom $=\operatorname{Hom}_{\mathbb{Z}}$.

Let "asterisk" denote applying $\operatorname{Hom}_{\mathbb{Z}}(-, \mathbb{Q} / \mathbb{Z})$. Duality homomorphisms (cf. [CE56, Prop. 5.4 of Ch. VI and Prop. 5.2' of Ch. II] - here the finiteness hypothesis on $\mathrm{H}_{q}(N, \mathbb{Z})$ is used) together with $M^{*} \cong M$, give us isomorphisms as horizontal arrows in the two subsequent diagrams

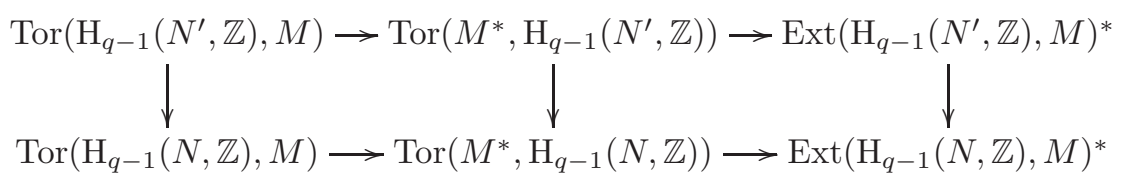

and

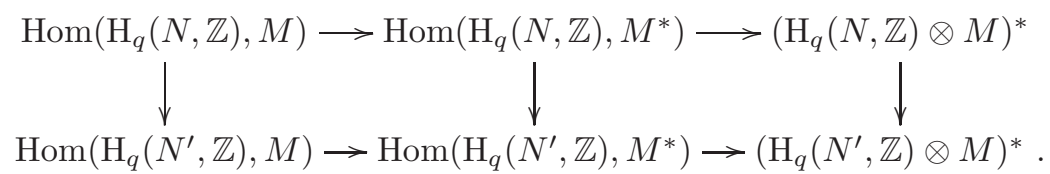

Since the restriction is null, it follows that so is the corestriction.

We mention that both reverse implications of the previous Lemma hold. The finiteness hypothesis in item (ii) is satisfied for groups of type FP over $\mathbb{Z}$.

Proof of Theorem F. Let $G$ be the profinite completion $\widehat{\Gamma}$ of a limit group $\Gamma$.

(a) Let $d=c d(\Gamma)$ and let $0 \rightarrow R_{d} \rightarrow \ldots \rightarrow R_{0} \rightarrow \mathbb{Z} \rightarrow 0$ be a projective resolution of the trivial $\mathbb{Z}[\Gamma]$-module $\mathbb{Z}$ with all $R_{i}$ finitely generated. Consider the finitely generated profinite projective $\mathbb{F}_{p} \llbracket \widehat{\Gamma} \rrbracket$-modules $P_{i}$ defined by $P_{i}=R_{i} \otimes_{\mathbb{Z}[\Gamma]} \mathbb{F} p \llbracket \widehat{\Gamma} \rrbracket$. In 
light of Lemma 4.1, the sequence $\ldots \rightarrow P_{2} \rightarrow P_{1} \rightarrow P_{0} \rightarrow \mathbb{F}_{p} \rightarrow 0$ is exact, since its $q$-th homology is isomorphic to $\varliminf_{N} \mathrm{H}_{q}\left(N, \mathbb{F}_{p}\right)$ (cf. [KZ08, Lemma 2.1]). So $G$ is of type $\mathrm{FP}_{\infty}$ over $\mathbb{F}_{p}$. Finally, since $\mathbb{Z}_{p}=\lim _{\longleftarrow} \mathbb{Z} / p^{k} \mathbb{Z}$, by passing to the limit it follows that $G$ is of type $\mathrm{FP}_{\infty}$ over $\mathbb{Z}_{p}$ (cf. [KZ08, Thm. 2.5]).

(b) Using the notation in item (a), consider the finitely generated profinite projetive $\mathbb{Z}_{p} \llbracket \widehat{\Gamma} \rrbracket$-modules $Q_{i}$ defined by $Q_{i}=R_{i} \otimes_{\mathbb{Z}[\Gamma]} \mathbb{Z}_{p} \llbracket \widehat{\Gamma} \rrbracket$. We have that $Q_{i} \otimes_{\mathbb{Z}_{p} \llbracket \widehat{\Gamma} \rrbracket} \mathbb{Z}_{p}$ is isomorphic to $R_{i} \otimes_{\mathbb{Z}[\Gamma]} \mathbb{Z}_{p}$ and hence to $R_{i} \otimes_{\mathbb{Z}_{p}[\Gamma]} \mathbb{Z} \otimes_{\mathbb{Z}} \mathbb{Z}_{p}$; thus its $\mathbb{Z}_{p}$-rank equals $\operatorname{rk}_{\mathbb{Z}}\left(R_{i} \otimes_{\mathbb{Z}[\Gamma]} \mathbb{Z}\right)$. So, the desired Euler-Poincaré characteristic over $\mathbb{Z}_{p}$ can be expressed as follows

$$
\begin{aligned}
\chi_{\mathbb{Z}_{p}}(\widehat{\Gamma}) & =\sum_{i}(-1)^{i} \operatorname{rk}_{\mathbb{Z}_{p}} \mathrm{H}_{i}\left(\widehat{\Gamma}, \mathbb{Z}_{p}\right)=\sum_{i}(-1)^{i} \operatorname{rk}_{\mathbb{Z}_{p}} \operatorname{Tor}_{i}^{\mathbb{Z}_{p} \llbracket \widehat{\Gamma} \rrbracket}\left(\mathbb{Z}_{p}, \mathbb{Z}_{p}\right) \\
& =\sum_{i}(-1)^{i} \operatorname{rk}_{\mathbb{Z}_{p}}\left(Q_{i} \otimes_{\mathbb{Z}_{p} \llbracket \widehat{\Gamma} \rrbracket} \mathbb{Z}_{p}\right)=\sum_{i}(-1)^{i} \operatorname{rk}_{\mathbb{Z}}\left(R_{i} \otimes_{\mathbb{Z}[\Gamma]} \mathbb{Z}\right) \\
& =\sum_{i}(-1)^{i} \operatorname{rk}_{\mathbb{Z}} \operatorname{Tor}_{i}^{\mathbb{Z}[\Gamma]}(\mathbb{Z}, \mathbb{Z})=\sum_{i}(-1)^{i} \operatorname{rk}_{\mathbb{Z}} \mathrm{H}_{i}(\Gamma, \mathbb{Z})=\chi(\Gamma) .
\end{aligned}
$$

The last assertion of item (b) follows from its discrete version proved by Kochloukova [Koch10, Lemma 5]. Note that the cohomological Euler-Poincaré characteristic of $\widehat{\Gamma}$ over $\mathbb{F}_{p}$ equals $\chi_{\mathbb{Z}_{p}}(\widehat{\Gamma})$ because

$$
\operatorname{dim}_{\mathbb{F}_{p}}\left(S_{i} \otimes_{\mathbb{F}_{p} \llbracket \widehat{\Gamma} \rrbracket} \mathbb{F}_{p}\right)=\operatorname{rk}_{\mathbb{Z}_{p}}\left(S_{i} \otimes_{\mathbb{Z}_{p} \llbracket \widehat{\Gamma} \rrbracket} \mathbb{Z}_{p}\right) .
$$

(c) Fix an index $k$. By Pontryagin duality, $\mathrm{H}_{q}\left(U_{k}, \mathbb{F}_{p}\right) \cong \mathrm{H}^{q}\left(U_{k}, \mathbb{F}_{p}^{*}\right)^{*}(c f$. [RZ00b, Prop. 6.3.6]). Moreover, $U_{k}$ is of type $\mathrm{FP}_{\infty}$ over $\mathbb{Z}_{p}$, because so is $G$; hence $\mathrm{H}^{q}\left(U_{k}, \mathbb{F}_{p}\right)$ is finite, and therefore $\mathrm{H}_{q}\left(U_{k}, \mathbb{F}_{p}\right)$ is isomorphic to $\mathrm{H}^{q}\left(U_{k}, \mathbb{F}_{p}\right)$. Now, let $N_{k}$ be the preimage of $U_{k}$ under the canonical map $\Gamma \rightarrow \widehat{\Gamma}$. Note that $\operatorname{dim} \mathrm{H}^{q}\left(U_{k}, \mathbb{F}_{p}\right)=\operatorname{dim} \mathrm{H}_{q}\left(N_{k}, \mathbb{F}_{p}\right)$. For, from goodness, $\mathrm{H}^{q}\left(U_{k}, \mathbb{F}_{p}\right) \cong \mathrm{H}^{q}\left(N_{k}, \mathbb{F}_{p}\right)$, and by the Universal coefficient theorem, $\mathrm{H}^{q}\left(N_{k}, \mathbb{F}_{p}\right) \cong \operatorname{Hom}\left(\mathrm{H}_{q}\left(N_{k}, \mathbb{F}_{p}\right), \mathbb{F}_{p}\right)$. So, the desired result follows from its discrete version demonstrated by Bridson and Kochloukova [BK17, Cor. B].

The rest of this section is devoted to the group-theoretic structure of groups in the class $\mathcal{Z}$. To prove Theorem B we show next that a very strong form of Tits alternative holds for these groups.

As generally expected, the profinite situation is more complex than discrete or pro- $p$. For instance, this is already evident in the class $\mathcal{Z}_{0}$ : it is easy to find a projective profinite group which is non-free, non-abelian, and virtually $\widehat{\mathbb{Z}}$ (e.g., some $\mathbb{Z}_{p^{\prime}} \rtimes \mathbb{Z}_{p}$ ). We have included a proof of the following straightforward lemma for the sake of convenience.

Lemma 4.2. Let $G$ be a projective profinite group. The following assertions are equivalent:

(i) G has no non-abelian free pro-p closed subgroup, for any prime $p$; 
(ii) $G$ is meta-procyclic projective (i.e., isomorphic to $\mathbb{Z}_{\pi} \rtimes \mathbb{Z}_{\rho}$, for some disjoint sets of primes $\pi$ and $\rho)$;

Moreover, if $G$ is nilpotent then it is procyclic.

Proof. From condition (i), every $p$-Sylow subgroup of $G$ is procyclic. Clearly, the $p$-Sylow subgroups of each finite quotient of $G$ are cyclic. Hence the commutator subgroup and the abelianization of each finite quotient of $G$ are cyclic groups of coprime orders (cf. [Zas49, Ch. V, Thm. 11]). Passing to the limit we get that $\overline{[G, G]}$ and $G / \overline{[G, G]}$ are procyclic groups of coprime orders. Thus, we reach (ii) from the profinite version of Schur-Zassenhaus theorem for profinite groups ( $c f$. [RZ00b, Thm. 2.3.15]).

Moreover, if $G$ is nilpotent, then it is the cartesian product of its Sylow subgroups, hence procyclic.

Theorem 4.3. Let $H$ be a closed subgroup of a group in the class $\mathcal{Z}$. The following assertions are equivalent:

(i) H has no non-abelian free pro-p closed subgroup, for any prime $p$;

(ii) $H$ is abelian or meta-procyclic projective;

Moreover, nilpotent closed subgroups of a group in the class $\mathcal{Z}$ are abelian, and there exists a uniform (finite) bound for their rank.

Proof. Say $H$ is a closed subgroup of some group $G_{n}$ of $\mathcal{Z}_{n}$. If $n=0$, then Lemma 4.2 concludes the proof.

Suppose then $n \geq 1$. Let $T$ be the standard tree on which $G_{n}$ acts, and restrict this action to $H$. From Lemma 3.3, the only possible cases of Theorem 2.2 are (a) and (c.1). This completes the result.

In particular we have proved Theorem B.

Corollary 4.4. The profinite completion of a non-abelian limit group has trivial centre.

Proof. Let $G$ be the profinite completion of a non-abelian limit group. If the nonabelian group $G$ is free profinite of finite rank, then it has trivial centre $Z(G)$ (cf. [RZ00b, Lemma 8.7.3(a)]).

Next, suppose $G$ is a closed subgroup of $G_{n}=\widehat{\Gamma_{n-1}} \amalg_{\widehat{Z_{n-1}}} \widehat{A_{n-1}}$, where $\Gamma_{n-1}$, $A_{n-1}$, and $Z_{n-1}$ are as in the proof of Theorem 3.4. Let $T$ be the standard profinite tree on which $G_{n}$ acts, and let $D$ be a minimal $Z(G)$-invariant profinite subtree of $T$ (see Proposition 2.3).

If $D$ is a singleton, then $Z(G)$ is the stabilizer of a vertex of $T$; in other words, $Z(G)$ is a subgroup of $g \widehat{\Gamma_{n-1}} g^{-1}$ or $g \widehat{A_{n-1}} g^{-1}$ for some $g$ in $G_{n}$. In the first situation we use induction on $n$; in the second, since $G$ is non-abelian, we obtain $Z(G)=$ 
$h^{-1} Z(G) h \subseteq \widehat{A_{n-1}} \cap h g \widehat{A_{n-1}}(h g)^{-1}$ for any $h$ in $G-\widehat{A_{n-1}}$. So, by Lemma 3.3, we get $Z(G)=\{1\}$.

Otherwise, by Lemma 3.3, we could apply [Zal90, Lemma 2.4(d)] to see that $Z(G)$ would act freely on $D$ and be isomorphic to $\mathbb{Z}_{\pi}$ for a non-empty set $\pi$ of primes; moreover, it would act freely on $T$ (cf. [ZM88, Thm. 2.13]). Now, writing $G=\widehat{\Gamma}$, we may and do assume that $\Gamma$ intersects some edge stabilizer of the tree on which it acts; for, otherwise $\Gamma$ is a free product, and hence $G$ is a free profinite product and thus a centreless group (cf. [RZ00b, Thm. 9.1.12] or [ZM88, Thm. 2.13]). Thus, the intersection of $G$ with a conjugate of $\widehat{Z_{n-1}}$ is isomorphic to $\widehat{\mathbb{Z}}$. Therefore, the closed subgroup $H$ of $G$ generated by $Z(G)$ and that intersection would be isomorphic to $\mathbb{Z}_{\pi} \times \widehat{\mathbb{Z}}$; hence $H$ would contain a closed subgroup isomorphic to $\mathbb{Z}_{p} \times \mathbb{Z}_{p}$ which, by Lemma 3.3 and Theorem 2.2, would fix a vertex of $T$. By [Zal90, Prop. 2.1], the minimal $H$-invariant profinite subtree of $T$ would be a vertex. So, this case cannot happen.

Now we turn to Theorem C. It follows at once from the next more general theorem, which should be compared with [HZZ16, Prop. 2.4], [SZ14, Thm. 3.5], and [WZ17a, Thm. 11.1].

Lemma 4.5 (cf. [WZ17b, Lemma 3.7]). Let $H$ be a pro-p group acting on a pro-p tree $T$ with compact non-empty edge set having only finitely many maximal vertex stabilizers up to conjugation. Suppose the distance between any two distinct vertices in the orbits of those finitely many vertices is infinite. If $k$ is a given integer $\geq 0$, then $H$ acts on a quotient pro-p tree $D$ with non-empty edge set such that the stabilizer of each edge in $D$ fixes at least $k$ edges in $T$.

Proof. Let $H_{v_{1}}, \ldots, H_{v_{n}}$ be maximal vertex stabilizers of $T$ up to conjugation; each edge or vertex $m$ of $T$ has stabilizer $H_{m}$ contained in some of $H_{v_{i}}$ up to $H$-translation of $m$. Assume $H_{m} \subseteq H_{v_{i}}$. Thus $H_{m}$ fixes the smallest pro- $p$ tree $S$ containing $m$ and $v_{i}$; if $m \neq v_{i}$, then each vertex of $S$ has an incident edge ( $c f$. [ZM88, Prop. 2.15]). Let $T_{k}(v)$ be a maximal subtree of radius $k$ around $v$. Thus, if $m \neq v_{i}$, then $m \notin T_{k}\left(v_{i}\right)$ and the stabilizer $H_{m}$ fixes at least $k$ distinct edges. Now $\bigcup_{i=1}^{n} H T_{k}\left(v_{i}\right)$ is a profinite $H$-subgraph of $T$ and collapsing all its connected components to distinct points in $T$ we obtain, by [Zal92, Proposition, p. 486], a pro- $p$ tree $D$ on which $H$ acts with edge stabilizers stabilizing at least $k$ distinct edges of $T$.

Theorem 4.6. Let $G$ be a profinite group acting acylindrically on an infinite profinite tree $T$ with compact edge set such that the edge stabilizers are procyclic. Each finitely generated pro-p closed subgroup $H$ of $G$ splits as

$$
H=\left(H \cap \operatorname{stab}_{G}\left(v_{1}\right)\right) \amalg^{p} \ldots \amalg^{p}\left(H \cap \operatorname{stab}_{G}\left(v_{n}\right)\right) \amalg^{p} F,
$$


where $\amalg^{p}$ denotes the free pro-p product, $v_{1}, \ldots, v_{n}$ are vertices of $T$, and $F$ is a free pro-p group.

Proof. By [SZ14, Thm. 3.5], there are only finitely many maximal by inclusion vertex stabilizers in $H$ up to conjugation. By [WZ17a, Lemma 11.2], we can apply Lemma 4.5 to see that $H$ acts on a profinite tree $D$ such that each edge stabilizer stabilizes at least $k$ distinct edges and hence is trivial. Then the result follows from [HZZ16, Prop. 2.4].

This proves Theorem C. We should mention that, by Lemma 3.3, the previous theorem follows by direct application of [WZ17b, Thm 3.8].

Finally we investigate the strong property of the transitivity of the commutation. The conclusion is Theorem D. Recall that a group $G$ is commutativity-transitive if for any three non-trivial elements $a, b$, and $c$ in $G$ if $a$ commutes with $b$ and $b$ commutes with $c$ then $a$ commutes with $c$; or equivalently, if the centralizer of each non-trivial element in $G$ is abelian.

Proposition 4.7. Let $H$ be a closed subgroup of a group in the class $\mathcal{Z}$, and let $g$ be an element of $H$ such that $\overline{\langle g\rangle}$ is isomorphic to $\widehat{\mathbb{Z}}$. Then:

(i) the centralizer $C_{H}(g)$ is maximal abelian or meta-procyclic projective;

(ii) the normalizer $N_{H}(\overline{\langle g\rangle})$ is maximal abelian or meta-procyclic projective, it is abelian only if it coincides with $C_{G}(g)$.

Proof. (i) It suffices to prove the result for $H=G_{n}$. We proceed by induction on $n$. By simplicity, set $G=G_{n}$ and $C=C_{G}(g)$.

If $n=0$, we have that $\overline{\langle g\rangle}$ is a normal subgroup of the projective profinite group $C$. Let $S$ be a $p$-Sylow subgroup of $C$. Then $\overline{\langle g\rangle} \cap S$ is the $p$-Sylow subgroup of $\overline{\langle g\rangle}$, and the free pro- $p$ group $S$ has a non-trivial normal procyclic subgroup; so $S$ is isomorphic to $\mathbb{Z}_{p}$. Hence $C$ is meta-procyclic projective, by Lemma 4.2.

Suppose then $n \geq 1$. Let $T$ be the standard profinite tree on which $G_{n}$ acts. By Proposition 2.3 there exists a (non-empty) minimal $C$-invariant profinite subtree $D$. When $D$ has only one element, there is an element $x$ in $G_{n}$ such that the conjugate subgroup $x C x^{-1}$ is contained in $G_{n-1}$ or $A_{n-1}$. By induction hypothesis, it follows that $C$ is abelian or meta-procyclic. Suppose $D$ has more than one element. Thus, $C$ acts faithfully on $D$ by Lemma 3.3; hence it is projective by [Zal90, Lemma 2.4(d)]. The second paragraph of this proof gives the result.

(ii) Since the automorphism group Aut $(\overline{\langle g\rangle})$ is abelian, the group $N_{H}(\overline{\langle g\rangle})$ is metabelian-by-abelian, and thus abelian or meta-procyclic projective, by Theorem 4.3. The last assertion of the theorem is obvious.

Acylindricality shortened the proof but it is not essential to the result. 
Corollary 4.8. In a group in the class $\mathcal{Z}$, each closed subgroup having $\widehat{\mathbb{Z}}$ as a direct factor must be abelian.

For the next and last theorem of this section we recall concepts of covers of profinite groups. Let $\Phi(G)$ denote the Frattini subgroup of a profinite group $G$. We say that an epimorphism of profinite groups $\varphi: \widetilde{G} \rightarrow G$ is a projetive (resp. Frattini) cover if, $\widetilde{G}$ is projetive (resp. $\operatorname{ker}(\varphi) \subseteq \Phi(\widetilde{G}))$. By [RZ00b, Lemma 2.8.15] (cf. [FJ05, Prop. 22.6.1]), to each profinite group $G$ there exists an epimorphism $\widetilde{\varphi}: \widetilde{G} \rightarrow G$, which is projetive and Frattini. Such cover, which is unique up to isomorphism, is called the universal Frattini cover.

Theorem 4.9. A commutativity-transitive group in the class $\mathcal{Z}$ is either abelian or pro-p.

Proof. A commutativity-transitive group will be called a CT-group, for simplicity. Let $G$ be a finitely generated profinite subgroup of some $G_{n}$ with $G$ a CT-group. The proof is by induction on $n$.

If $n=0$, then $G$ is either procyclic or non-abelian free pro- $p$. Indeed, let $Q$ be a non-abelian finite quotient of $G$ having elements of distinct coprime orders. Then, the canonical map $G \rightarrow Q$ factors through the universal Frattini cover $\widetilde{\varphi}: \widetilde{Q} \rightarrow Q$ (since $G$ is projective), in such a way that $G \rightarrow \widetilde{Q}$ is also an epimorphism (because $\operatorname{ker}(\widetilde{\varphi}) \subseteq \Phi(\widetilde{Q}))$. Now, the Frattini subgroup $\Phi(\widetilde{Q})$ is an open normal subgroup of $\widetilde{Q}$, and $\widetilde{Q}$ has a non-trivial $p$-Sylow, say $S_{p}$. Intersecting $S_{p}$ with $\Phi(\widetilde{Q})$ we obtain a $p$-Sylow subgroup of $\Phi(\widetilde{Q})$, which has finite index in $S_{p}$. Thus, since $\Phi(\widetilde{Q})$ is pronilpotent, Sylow subgroups of $\widetilde{Q}$ relative to distinct primes must commute pointwise; hence $\widetilde{Q}$ is pronilpotent. On the other hand, being $\widetilde{Q}$ projetive, it can be seen as a subgroup of $G$; so $\widetilde{Q}$ is also a CT-group. Therefore, each Sylow subgroup of $\widetilde{Q}$ is abelian; hence so is $\widetilde{Q}$.

Next, assume the result is true for the class $\mathcal{Z}_{n-1}$. Consider $G_{n} \in \mathcal{Z}_{n}$ where $G_{n}=G_{n-1} \amalg_{C_{n-1}} A_{n-1}$ with $A_{n-1}=B_{n-1} \times C_{n-1}$ and $G_{n-1} \neq C_{n-1} \neq A_{n-1}$. Let $D_{n-1}$ be a non-trivial procyclic maximal direct factor of $B_{n-1}$ (which may coincide with $\left.B_{n-1}\right)$ and define $K=\overline{\left\langle g D_{n-1} g^{-1} \mid g \in G_{n}\right\rangle}$. By [Zal95, Thm. B], $K$ is a projective profinite group. Consider $H=K \cap G$. Since in the second paragraph of the proof we did not use the fact that $G$ is finitely generated, we have three possibilities for $H$ : non-abelian free pro- $p$, non-trivial procyclic, or trivial. To conclude the proof we henceforth suppose that $G$ is not pro- $p$.

First, we claim that $H$ cannot be a non-abelian free pro- $p$ group. Indeed, since there exists an element $y$ in $G$ such that $\overline{\langle y\rangle}$ is isomorphic to $\mathbb{Z}_{q}$ with $q \neq p$, and since $G$ is a CT-group, the subgroup $\overline{\langle y\rangle}$ would act non-trivially on the normal subgroup $H$; that is, there would exist $x$ in $H$ that would not commute with 
$y$. So the subgroup $\overline{\langle x, y\rangle}$ would be CT, projetive, non-abelian and non-pro- $p$; a contradiction to the second paragraph.

Second, suppose that $H$ is non-trivial procyclic. We take a non-trivial $\ell$-Sylow subgroup $S$ of $H$, which is characteristic in $H$, and hence normal in $G$. If $S$ is central in $G$, then $G$ must be abelian. Otherwise, there would exist an element in $G$ that would not commute with $S$ and hence there would exist a subgroup $T$, of order a power of a prime $r$, that would not commute with $S$. Consider then S.T, which would be isomorphic to $\mathbb{Z}_{\ell} \rtimes \mathbb{Z}_{r}$. If $r \neq \ell$, then this product, and hence $G$, would not be CT (because a non-abelian CT-group is centreless); the case $r=\ell$, could not happen by Theorem 4.3 .

Third, suppose $H$ is trivial. Note that, $K$ is the kernel of the epimorphism $G_{n} \longrightarrow G_{n-1} \amalg_{C_{n-1}}\left(A_{n-1} / D_{n-1}\right)$ induced by the identity map of $G_{n-1}$ and by the canonical map $A_{n-1} \rightarrow A_{n-1} / D_{n-1}$. Thus we have an embedding

$$
G \longrightarrow G_{n-1} \amalg_{C_{n-1}}\left(A_{n-1} / D_{n-1}\right) .
$$

If $d\left(A_{n-1}\right)=1$, then $A_{n-1} / D_{n-1}$ is $C_{n-1}$ and we are done; otherwise, $d\left(A_{n-1} / D_{n-1}\right)<d\left(A_{n-1}\right)$. So, the result follows inductively.

Corollary 4.10. The profinite completion of a limit group $\Gamma$ is commutativitytransitive if, and only if, $\Gamma$ is a free abelian group of finite rank.

Proof. Being $\Gamma$ residually free, if it is non-abelian, then it has a finite non- $p$-group as a quotient; hence its profinite completion is not a pro- $p$ group.

\section{Final Comments}

In this last section we make a few comparisons concerning group-theoretical properties of limit groups, "limit pro- $p$ groups" (i.e., those studied in [KZ11]), and groups in the class $\mathcal{Z}$

First, if $p$ and $q$ are distinct primes and $q$ divides $\max \{2, p-1\}$, consider $\mathbb{Z}_{p} \rtimes \mathbb{Z}_{q}$. This example shows that non-abelian groups in the class $\mathcal{Z}$ may have non-trivial centre (versus non-abelian limit groups must have trivial centre); moreover, virtually abelian groups in the class $\mathcal{Z}$ are not necessarily abelian (versus virtually nilpotent limit groups must be abelian).

Next, groups in $\mathcal{Z}$ may have finite continuous abelianization; e.g., the universal Frattini cover of a non-abelian finite simple group has trivial continuous abelianization (because any Frattini cover of a perfect profinite group is perfect).

Furthermore, the non-procyclic group $\widehat{\mathbb{Z}} \amalg \mathbb{Z}_{p}$ has deficiency one, while every closed abelian subgroup of it is at most 1-generated. For limit groups or "limit pro- $p$ groups", if every closed abelian subgroup at most 1-generated but the groups themselves are not 1-generated then their deficiency must be $\geq 2$.

Also, Howson's property fails for groups in the class $\mathcal{Z}$ already at $n=0$ : 
Proposition 5.1. The free profinite group of rank 2 does not satisfy the finitely generated intersection property of Howson.

Proof. (A. Jaikin-Zapirain [J15]). Let $p$ and $q$ be two different primes. Pick a closed normal subgroup $N$ of a free profinite group $F$ of rank 2 such that $F / N$ is isomorphic to the free pro- $q$ group $A$ with basis $\{a\}$. Let $H=N / N_{p}$ be the maximal pro- $p$ quotient of $N$. So, the 2-generated group $G=F / N_{p}$ is a semi-direct product of the infinitely generated free pro- $p$ group $H$ and $A$.

Now, let $T$ be a free pro- $q$ group with basis $\left\{g_{1}, g_{2}\right\}$. Define its action on $H$ in such a way that $g_{1}$ and $g_{2}$ act as does $a$. Form a semidirect product $T \ltimes H$ and, for $i=1,2$, take the subgroup $G_{i}$ topologically generated by $g_{i}$ and $H$ therein. Being $G_{1}$ and $G_{2}$ isomorphic to $G$, they are 2-generated.

So, $T \ltimes H$ is a 3 -generated profinite group. Since its Sylow subgroups are free, it has cohomological dimension 1 , and therefore it is a subgroup of a free profinite group. But $G_{1} \cap G_{2}=H$ is not finitely generated.

Finally, a non-abelian free profinite group cannot be residually soluble. We do not know whether the "limit pro- $p$ groups" are necessarily residually nilpotent.

\section{ACKNOWLEDGEMENTS}

Both authors are grateful for the partial financial support from $\mathrm{CNPq}$ and CAPES.

\section{REFERENCES}

[AB06] E. Alibegović and M. Bestvina, Limit groups are CAT(0), J. London Math. Soc. (2), 74 (2006), 259-272.

[BBau67] B. Baumslag, Residually free groups, Proc. London Math. Soc. (3), 17 (1967), 402-418.

[Bau62] G. Baumslag, On generalised free products, Math. Z., 78 (1962), 423-438.

[BH07] M. Bridson and J. Howie, Normalisers in limit groups, Math. Ann., 337 (2007), no. 2, 385-394.

[BK17] M. Bridson and D. Kochloukova, Volume gradients and homology in towers of residuallyfree groups, Math. Ann., 367 (2017), 1007-1045,

[CE56] H. Cartan and S. Eilenberg, Homological algebra, Princeton University Press, 1956, Princeton.

[CZ07] S. Chagas and P. Zalesskii, Limit Groups are conjugacy separable, Internat. J. Algebra Comput., 17 (2007), no. 4, 851-857.

[CG05] C. Champetier and V. Guirardel, Limit groups as limits of free groups, Israel J. Math., 146 (2005), 1-75.

[FJ05] M. Fried and M. Jarden, Field arithmetic, Springer-Verlag, New York, 2005.

[J15] A. Jaikin, Do free profinite groups satisfy Howson's theorem?, in MathOverflow, http://mathoverflow.net/questions/195166, accessed 2015-02-02.

[HZZ16] W. Herfort, P. Zalesskii, and T. Zapata, Splitting theorems for pro-p groups acting on pro-p trees, Selecta Math. (N.S.), 22, (2016), no. 3 1245-1268. http://dx.doi.org/10.1007/s00029-015-0217-7.

[KhM98a] O. Kharlampovich and A. Myasnikov, Irreducible affine varieties over a free group. I. Irreducibility of quadratic equations and Nullstellensatz., J. Algebra, 200 (1998), no. 2, $472-516$.

[KhM98b] O. Kharlampovich and A. Myasnikov, Irreducible affine varieties over a free group. II. Systems in triangular quasi-quadratic form and description of residually free groups, J. Algebra, 200 (1998), no. 2, 472-516. 
[KhM06] O. Kharlampovich and A. Myasnikov, Elementary theory of free non-abelian groups, J. Algebra, 302 (2006), no. 2, 451-552.

[Koch10] D. Kochloukova, On subdirect products of type $\mathrm{FP}_{m}$ of limit groups, J. Group Theory, 13 (2010), no. 1, 1-19.

[KZ08] D. Kochloukova and P. Zalesskii, Profinite and pro-p completions of Poincaré duality groups of dimension 3, Trans. Amer. Math. Soc., 360 (2008), no. 4, 1927-1949.

[KZ11] D. Kochloukova and P. Zalesskii, On pro-p analogues of limit groups via extensions of centralizers, Math. Z., 267 (2011), no. 1-2, 109-128.

[Rem89] V. Remeslennikov, $\exists$-free groups, Sibirsk. Mat. Zh., 30 (1989), 193-197.

[Rib71] L. Ribes, On amalgamated products of profinite groups, Math. Z., 123 (1971), 357-364.

[Rib17] L. Ribes, Profinite graphs and groups, Springer, 2017.

[RZ96] L. Ribes and P. Zalesskii, Conjugacy separability of amalgamated free products of groups, J. Algebra 179 (1996), no. 3, 751-774.

[RSZ96] L. Ribes, D. Segal, and P. Zalesskii, Conjugacy separability and free products of groups with cyclic amalgamation, J. London Math. Soc. (2) 57 (1998), no. 3, 609-628.

[RZ00a] L. Ribes and P. Zalesskii, Pro- $p$ trees and applications, New horizons in pro- $p$ groups, Progr. Math., 184, 75-119, Birkhäuser Boston, Boston, MA, 2000.

[RZ00b] L. Ribes and P. Zalesskii, Profinite groups, Springer-Verlag, Berlin, 2000.

[Sel01] Z. Sela, Diophantine geometry over groups. I. Makanin-Razborov diagrams, Publ. Math. Inst. Hautes Études Sci., 93 (2001), 31-105.

[Sel06] Z. Sela, Diophantine geometry over groups. VI. The elementary theory of a free group, Geom. Funct. Anal., 16 (2006), no. 3, 707-730.

[Ser77] J-P. Serre, Arbres, amalgames, SL 2 , Société Mathématique de France, Paris, 1977.

[Ser94] J-P. Serre, Cohomologie Galoisienne, Lecture Notes in Mathematics, 5, Springer-Verlag, Berlin, 1994

[SZ14] I. Snopce and P. Zalesskii, Subgroup properties of pro-p extensions of centralizers, Selecta Math. (N.S.), 20, (2014)

[Tit70] J. Tits, Sur le groupe des automorphismes d'un arbre. In Essays on topology and related topics (Mémoires dédiés à Georges de Rham), 188-211, Springer, New York, 1970.

[Wil08] H. Wilton, Hall's theorem for limit groups, Geom. Funct. Anal., 18 (2008), 271-303.

[WZ17a] H. Wilton and P. Zalesskii. Distinguishing geometries using finite quotients, Geom. Topol., 21(1), 345-384, 2017.

[WZ17b] H. Wilton and P. Zalesskii. Pro-p subgroups of profinite completions of 3-manifold groups 96, (2017), 293-308

[Zal89] P. Zalesskii, Geometric characterization of free constructions of profinite groups, Siberian Math. J., 30 (1989), no. 2, 227-235.

[Zal90] P. Zalesskii, Profinite groups, without free nonabelian pro-p-subgroups, that act on trees, Mat. Sb., 181 (1990), no. 1, 57-67.

[Zal92] P. Zalesskii, Open Subgroups of Free Profinite Products. In Proceedings of the International Conference on Algebra, Part 1 (Novosibirsk, 1989), 473-491, Contemp. Math., 131, Part 1, Amer. Math. Soc., Providence, RI, 1992.

[Za195] P. Zalesskii, Normal subgroups of free constructions of profinite groups and the congruence kernel in the case of positive characteristic, Izv. RAN. Ser. Mat., 59:3 (1995), 59-76.

[ZM88] P. Zalesskii and O. Mel'nikov, Subgroups of profinite groups acting on trees, Mat. Sb. (N.S.), 135(177) (1988), no. 4, 419-439, 559.

[ZM89] P. Zalesskii and O. Mel'nikov, Fundamental groups of graphs of profinite groups, Algebra i Analiz, 1 1989, no. 4, 117-135.

[Zap11] T. Zapata, Grupos pro-finitos limites, Ph.D thesis, Universidade de Brasília, 2011.

[Zas49] H. Zassenhaus, The Theory of Groups, Chelsea Publishing Company, New York, 1949.

May 15, 2018.

Department of Mathematics, University of Brasilia, Brazil

E-mail addresses: pz@mat.unb.br and zapata@mat.unb.br 